\title{
Place et rôle de l'ubiquitine dans le développement de l'auto-immunité au cours de la maladie lupique
}

Les anticorps anti-ubiquitine représentent l'un des meilleurs marqueurs actuellement connus du lupus érythémateux disséminé, s'ajoutant à d'autres anticorps antiprotéines du stress (les heat shock proteins) récemment décrits dans diverses affections. Les auto-anticorps de patients lupiques sont spécifiquement dirigés contre des protéines ubiquitinylées, notamment l'histone H2A, si bien que l'hypothèse peut être faite que l'ubiquitinylation de divers constituants cellulaires altérés par des processus variés (infection, toxiques, ultraviolets, etc.) est une étape importante du développement de l'autoimmunité chez des sujets génétiquement prédisposés.

\section{Sylviane Muller}

\section{ABRÉVIATIONS}

U-H2A : histone H2A ubiquitinylée HSP : heat shock proteins

HMG : high mobility group proteins

LED : lupus érythémateux disséminé PR : polyarthrite rhumatoïde

ACJ : arthrite chronique juvénile

ELISA : enzyme-linked immunosorbent assay

\section{ADRESSE}

S. Muller : docteur ès sciences, chargée de recherche au CNRS. Laboratoire d'immunochimie, Institut de biologie moléculaire et cellulaire du Cnrs, 15, rue René-Descartes, 67084 Strasbourg Cedex, France. es facteurs étiologiques impliqués dans l'autoimmunité non spécifique d'organes (lupus érythémateux disséminé, LED), polyarthrite rhumatoïde ou syndrome de Gougerot-Sjögren par exemple) sont très mal élucidés. Plusieurs théories ont été présentées qui, à elles seules cependant, ne permettent pas de réconcilier tous les faits cliniques et expérimentaux décrits. On insiste notamment sur l'existence d'un terrain immunitaire particulier hyperréactif où certains lymphocytes $B$, éventuellement des clones autoréactifs, pourraient jouer un rôle déterminant. Un déséquilibre des souspopulations lymphocytaires $\mathrm{T}$ pourrait également contribuer à l'état auto-immun. L'implication des protéines de stress dans différents mécanismes immunitaires a émergé depuis quelques années. L'une de ces pro- téines, l'ubiquitine, pourrait être impliquée dans l'induction d'autoanticorps et donc jouer un rôle important dans le LED. Parmi ses différentes fonctions, l'ubiquitine intervient dans la protéolyse intracellulaire de protéines altérées, dans la structure de la chromatine et du ribosome, et dans la capture des lymphocytes dans les tissus lymphoïdes. Selon les cellules considérées et leur phase au cours du cycle cellulaire, de 30 à plus de $80 \%$ de l'ubiquitine se présentent sous forme conjuguée à des protéines acceptrices très diverses. Chez les patients atteints de LED, des anticorps circulants antiubiquitine et en particulier des anticorps dirigés contre des complexes histone H2A-ubiquitine ont été mis en évidence. Ces auto-anticorps sont, à l'instar des anticorps anti-ADN, des marqueurs très caractéristiques de la maladie lupique. Leur origine n'est 


\section{RÉFÉRENCES}

1. Morrow J, Isenberg D. Auto-immune Rheumatic Disease. Oxford : Blackwell Scientific Publications, 1987 : 324 p.

2. Morange $\mathrm{M}$, Bensaude O. Protéines de stress et immunité. Ann Inst Pasteur/Actualités $1991 ; 2$ : 51-9.

3. Rechsteiner M. Ubiquitin. New York : Plenum Press, 1988 : $346 \mathrm{p}$

4. Goldstein G, Scheid M, Hammerling U, Boyre EA, Schlesinger DH, Niall HD. Isolation of a polypeptide that has lymphocytedifferentiating properties and is probably represented universally in living cells. Proc Natl Acad Sci USA 1975 ; 72 : 11-5.

5. Hershko A, Ciechanover A, Heller $H$, Haas AL, Rose IA. Proposed role of ATP in protein breakdown : conjugation of proteins with multiple chains of the polypeptide of ATP-dependent proteolysis. Proc Nat Acad Sci USA 1980 ; 77 : 1783-6.

6. Vijay-Kumar S, Bugg CE, Cook WJ Structure of ubiquitin refined at 1.8 A resolution. $J$ Mol Biol 1987 ; 194 : 531-44.

7. Hershko A. Ubiquitin-mediated protein degradation. J Biol Chem $1988 ; 263$ : 15237-40.

8. Kudo M, Sugasawa K, Hori TA, Enomoto T, Hanaoka F, Ui M. Human ubiquitin-activating enzyme (E1) : compensation of heat-labile mouse $\mathrm{E} 1$ and its gene localization on the X-chromosome. Exp Cell Res 1991; 192: 110-7.

9. Fried VA, Smith HA, Hildebrandt E, Weiner K. Ubiquitin has intrinsic proteolytic activity : implications for cellular regulation. Proc Natl Acad Sci USA 1987 ; 84 : 3685-9.

10. Briand JP, Van Dorsselaer A, Raboy B, Muller S. Total chemical synthesis of ubiquitin using BOP reagent : biochemical and immunochemical properties of the purified product. Peptide Res 1989; 2 : 381-8.

11. Ciechanover A, Schwartz AL. How are substrates recognized by the ubiquitinmediated proteolytic system ? Trends Biochem Sci 1989 ; 14 : 483-8.

12. Thorne $A W$, Sautière $P$, Briand $G$, Crane-Robinson C. The structure of ubiquitinated histone H2B. EMBO J $1987 ; 6$ : 1005-10.

13. Siegelman $\mathbf{M}$, Bond $\mathbf{M W}$, Gallatin WM, et al. Cell surface molecule associated with lymphocyte homing is a ubiquitinated branched-chain glycoprotein. Science 1986 ; pas connue, mais l'on peut suggérer qu'elle soit dépendante, dans un contexte génétique prédisposé, d'un mécanisme où les événements de mort cellulaire (apoptose) joueraient un rôle déterminant.

\section{Introduction}

Aux États-Unis, de 500000 à 1 million de personnes souffrent de lupus érythémateux disséminé (LED) et 50000 nouveaux cas y sont rapportés chaque année. Le LED est un désordre immunologique chronique systémique atteignant principalement la femme avant 40 ans et qui se caractérise par la production d'autoanticorps, en particulier des anticorps dirigés contre des antigènes nucléaires. Ces auto-anticorps s'associent au développement de lésions tissulaires mettant en jeu des réactions immunitaires [1]. La gravité de la maladie est liée à l'atteinte de tissus vitaux, tels les reins et le système nerveux central, et aux complications, en particulier infectieuses, liées aux traitements agressifs parfois nécessaires pour contrôler les symptômes de la maladie.

L'étiologie et la physiopathologie de cette maladie restent mal connues. En particulier, comme dans tous les cas de maladies non spécifiques d'organes, on connaît très mal la nature des processus mis en jeu lors du déclenchement de l'atteinte, qui est extrêmement hétérogène. De même, les mécanismes lésionnels et la pathogénie de ces maladies demeurent très mal compris. Par le qualificatif d'atteinte multifactorielle, on accorde à ces maladies auto-immunes des causes relevant à la fois de facteurs immunologiques, génétiques, endocriniens et environnementaux qui se combinent de façons diverses et s'expriment de manière très polymorphique chez les patients. Ces inconnues rendent compte du fait que le traitement reste en pratique essentiellement empirique.

Ces quelques données liées au LED illustrent l'importance que va revêtir la qualité du diagnostic. Le traitement appliqué sera d'autant plus efficace que le diagnostic aura été établi précocement. Depuis quelques années, des développements techniques très intéressants (nouvelles approches biochimiques et de biologie moléculaire) ont permis une meilleure caractérisation des autoanticorps et de leur(s) cible(s), ce qui autorise aujourd'hui à reconsidérer certaines hypothèses touchant l'ensemble des phénomènes pathologiques auto-immuns. Les protéines de stress (ou protéines de choc thermique, HSP) sont notamment apparues comme des éléments importants susceptibles de jouer un rôle majeur dans divers mécanismes immunitaires [2]. Parmi la grande famille très conservée des HSP, on distingue plusieurs groupes rassemblant ces protéines selon leur masse moléculaire. L'ubiquitine, sur laquelle nous allons centrer cette revue, appartient à un groupe où elle est l'unique représentante. C'est une petit molécule "surdouée" qui intervient à toutes les phases du cycle cellulaire et dont l'ubiquité cellulaire et fonctionnelle remarquable lui donne une position stratégique dans le champ des recherches sur certains phénomènes autoimmuns $\left(\mathrm{m} / \mathrm{s} n^{\circ} 5\right.$, vol. 2, p. 282 et $n^{\circ} 1$, vol. 4, p. 59). La découverte récente d'auto-anticorps antiubiquitine et surtout anti-protéines nucléaires ubiquitinylées au cours du développement de maladies autoimmunes ouvre des perspectives nouvelles. En particulier, cette découverte repose le problème des liens existant entre, d'une part, des facteurs externes ou internes perturbant la cellule et, d'autre part, l'auto-immunité. Dans un contexte immunitaire particulier hyperréactif, les modifications post-traductionnelles normalement transitoires des protéines, et dont certaines sont associées aux éléments du système de réparation cellulaire, pourraient jouer un rôle dans le déclenchement des phénomènes autoimmunitaires.

\section{Structure de I'ubiquitine}

L'ubiquitine est une molécule de 76 résidus d'acides aminés et de poids moléculaire 8565 , présente dans les cellules de tous les organismes eucaryotes [3]. Elle existe sous des formes très variées, libre ou conjuguée à des accepteurs très divers, nucléaires, cytoplasmiques ou membranaires. Elle est extrêmement con- 


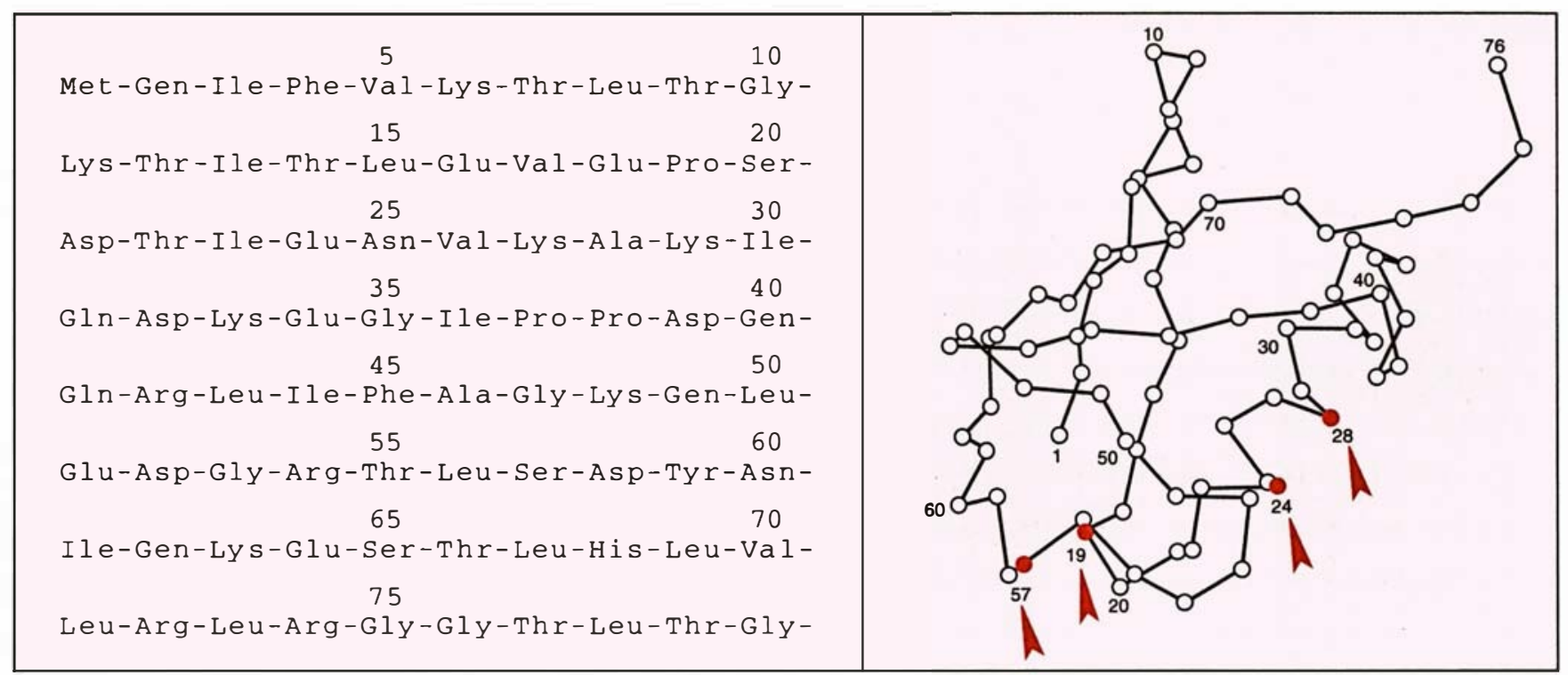

Figure 1. Structure de l'ubiquitine. Les flèches indiquent les sites connus de substitution de résidus d'acides aminés. (D'après [6].)

servée depuis l'amibe jusqu'à l'homme. Seules quatre positions présentent des variations, à savoir les résidus $19,24,28$ et 57 . Ces substitutions sont conservatives et lorsque la structure tridimensionnelle de l'ubiquitine a été déterminée, il s'est avéré que ces quatre sites étaient très proches dans la configuration spatiale de la molécule (figure 1). L'ubiquitine est une protéine étonnamment stable qui peut être portée à ébullition ou placée à $\mathrm{pH} 2$ ou 9 et retrouver son activité ; elle est très soluble en phase aqueuse. Elle est généralement très résistante aux protéases, mais elle est inactivée par la trypsine; elle n'est pas glycosylée et ne renferme pas de résidus de cystéine ou de tryptophane. Elle est globalement neutre (point iso-électrique : 6,7), renferme 11 résidus basiques, 11 résidus acides et un seul résidu d'histidine. En dehors d'une séquence proline-proline (résidus 37 et 38), elle ne présente aucun élément particulier dans sa structure primaire.

L'abondance intracellulaire de l'ubiquitine est remarquable puisqu'elle approche celle de l'actine, de la tubuline ou des histones, et il est surprenant que la découverte de cette molécule soit aussi récente. Elle a en effet été isolée en 1975 de manière fortuite par Goldstein et ses collaborateurs [4]

$\mathrm{m} / \mathrm{s} n^{\circ} 3$, vol. 8 , mars 92 qui lui attribuèrent, à l'époque, des rôles hormonaux dans l'induction de la différenciation de lymphocytes $\mathrm{B}$ et l'activation de l'adénylate cyclase, rôles qui furent démentis par la suite. On décrivait alors une molécule inactive de 74 résidus et ce n'est qu'en 1980 qu'on découvrit que les deux résidus glycine-glycine en position 75 et 76 C-terminale, facilement clivés lors des étapes de purification, étaient responsables de son attachement aux divers accepteurs et entraient dans un schéma réactionnel central de l'ubiquitine [5].

La structure cristallographique de l'ubiquitine est connue à $1,8 \AA$ (figure 1). Elle est très globulaire, avec un corps central très compact et hydrophobe responsable de sa grande stabilité. Elle présente une hélice $\alpha$ et quatre feuillets $\beta$. A l'extrémité Cterminale, quatre résidus sont particulièrement exposés à la surface de la molécule. Ce sont ces résidus et notamment les résidus glycine-glycine qui sont clivés par la trypsine en entraînant l'inactivation de l'ubiquitine.

\section{Gènes codant pour l'ubiquitine}

L'organisation des gènes codant pour l'ubiquitine se présente sous deux modes : d'une part, divers auteurs ont montré l'existence de polygènes de longueurs variables codant pour 3 à plus de 15 gènes d'ubiquitine arrangés dans un système tête-bêche sans séquence intermédiaire et qui se terminent par une séquence codante dans laquelle la glycine C-terminale traduite est bloquée par un résidu additionnel [3]. La longueur de ces gènes de polyubiquitine, le nombre de loci et l'acide aminé bloquant le résidu C-terminal varient en fonction des espèces. Par ailleurs, plus récemment, un deuxième mode d'organisation a été mis en évidence dans lequel un groupe de gènes codant pour une seule copie d'ubiquitine est fusionnée à une protéine dite d'extension de 52 ou 76 à 80 résidus. $\mathrm{Ce}$ polypeptide d'extension C-terminal additionnel est très conservé. Il possède un motif typique présent, par exemple, dans le facteur de transcription TF III A, et caractérisé par un arrangement en "doigt de zinc " supposé impliqué dans la régulation de divers gènes. La fonction de cette protéine de fusion (CEP) n'a pas encore été définie clairement. On sait cependant que la CEP 80 est spécifiquement associée à la sous-unité ribosomale $40 \mathrm{~S}$ de monocytes humains et correspond à la protéine S27a. La CEP 52 a été identifiée comme une 


\section{RÉFÉRENCES}

14. Mueller RD, Yasuda $\mathrm{H}$, Hatch $\mathrm{CL}$ Bonner WM, Bradbury EM. Identification of ubiquitinated histones $2 \mathrm{~A}$ and $2 \mathrm{~B}$ in Physarum Polycephalum : disappearance of these proteins at metaphase and reappearance at anaphase. $J$ Biol Chem $1985 ; 260$. 5147-53.

15. Monia BP, Ecker DJ, Crooke ST. New perspectives on the structure and function of ubiquitin. Biotechnology $1990 ; 8$ : 209-15.

16. Tabaton $\mathbf{M}$, Cammarata S, Mancardi $\mathrm{G}$, et al. Ultrastructural localization of $\beta$ amyloid, $\tau$, and ubiquitin epitopes in extracellular neurofibrillary tangles. Proc Natl Acad Sci USA 1991; 88 : 2098-102.

17. Mayer RJ, Lowe J, Landon M. Ubiquitin and the molecular pathology of chronic degenerative diseases. J Pathol 1991 ; $163: 279-81$

18. Muller S, Bonnier D, Thiry M, Van Regenmortel MHV. Reactivity of autoantibodies in systemic lupus erythematosus with core histone synthetic peptides. Int Arch All Appl Immunol 1989 ; 89 : 288-96.

19. Tuaillon $\mathrm{N}$, Muller S, Pasquali JL, Bordigoni P, Youinou P, Van Regenmortel MHV. Antibodies from patients with rheumatoid arthritis and juvenile rheumatoid arthritis analyzed with core histone synthetic peptides. Int Arch All Appl Immunol $1990 ; 91$ : 297-305.

20. Barakat S, Briand JP, Weber JC, Van Regenmortel MHV, Muller S. Recognition of synthetic peptides of Sm-D auto-antigen by lupus sera. Clin Exp Immunol 1990 ; 81 : 256-62.

21. Barakat S, Briand JP, Abuaf N, Van Regenmortel MHV, Muller S. Mapping of epitopes on U1 snRNP polypeptide A with synthetic peptides and autoimmune sera. Clin Exp Immunol 1991; 86 : 71-8.

22. Muller S, Briand JP, Van Regenmortel MHV. Presence of antibodies to ubiquitin during the autoimmune response associated with systemic lupus erythematosus Proc Natl Acad Sci USA 1988 ; 85 : 1176-80.

23. Muller S, Barakat S, Watts R, Joubaud $P$, Isenberg DA. Longitudinal analysis of antibodies to histones, Sm-D peptides and ubiquitin in the serum of patients with systemic lupus erythematosus, rheumatoid arthritis and tuberculosis. Clin Exp Rheum 1990 ; $8: 445-53$.

24. Plaué S, Muller S, Van Regenmortel MHV. A branched synthetic octapeptide of ubiquitinated histone $\mathrm{H} 2 \mathrm{~A}$ as target of autoantibodies. $J$ Exp Med $1989 ; 169$ : nouvelle protéine ribosomale de la sous-unité $60 \mathrm{~S}$ dans la levure et chez l'homme.

\section{Fonctions de l'ubiquitine}

L'ubiquitine intervient dans les principales étapes du métabolisme cellulaire $\left(\mathrm{m} / \mathrm{s} n^{\circ} 5\right.$, vol. 2, p. 282 et $n^{\circ} 1$, vol. 4, p. 59) (figure 2). L'une des fonctions de l'ubiquitine - certainement la plus étudiée - concerne son implication dans la dégradation protéolytique non lysosomiale ainsi que lysosomiale de protéines altérées ou de durée de vie courte [7]. Pour éliminer les protéines destinées au catabolisme, l'ubiquitine se lie de manière covalente à la protéine cible par l'intermédiaire d'une liaison peptidique entre le groupe carboxylique de son résidu glycine C-terminal (position 76) et un groupe aminé de la protéine, soit en position $\alpha$ $\mathrm{NH}_{2}$-terminale libre, soit au niveau du $\epsilon-\mathrm{NH}_{2}$ d'un résidu de lysine interne. Cette liaison nécessite la présence d'ATP et un système multienzymatique qui, dans les réticulocytes, contient au moins sept protéines différentes. Les principales étapes sont résumées sur la figure 3 .
Dans ce schéma, l'enzyme E1 joue le rôle d'activateur de l'ubiquitine. Récemment, le gène fonctionnel codant pour l'enzyme E1 humaine a été localisé sur le chromosome $\mathrm{X}$ [8]. Une protéine cible peut être multiubiquitinylée par attachement covalent d'un chapelet de molécules d'ubiquitine liées entre elles au niveau de la lysine 48 de chaque ubiquitine. Une fois la protéine "marquée " par une ou plusieurs molécules d'ubiquitine, le conjugué est dégradé par un système de protéases relativement peu spécifiques en libérant l'ubiquitine intacte. Fried et al. [9] ont montré que l'ubiquitine elle-même pourrait posséder une certaine activité protéolytique, mais ce résultat n'a pu être reproduit ultérieurement [10]. Un deuxième mécanisme a été décrit dans lequel une isopeptidase désassemble le conjugué en libérant l'ubiquitine de la protéine cible. $\mathrm{Ce}$ schéma réactionnel correspondrait soit à un système de sauvegarde empêchant une protéine d'être protéolysée à tort, soit à une voie de régulation du taux d'ubiquitinylation de protéines spécifiques, soit enfin à un moyen d'élimination des fragments protéolytiques engendrés pendant la digestion des conjugués à partir de l'extrémité

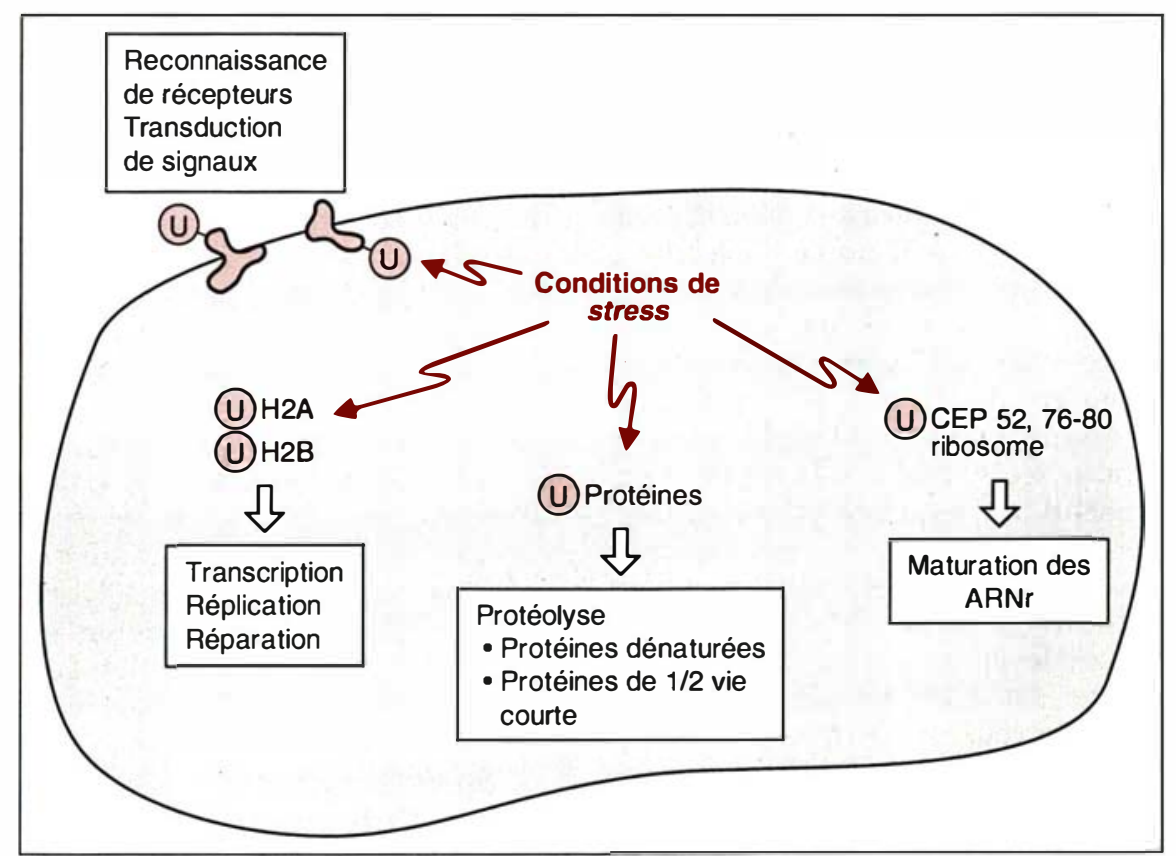

Figure 2. Rôles supposés de l'ubiquitine dans le métabolisme cellulaire. $U$ : ubiquitinylation. 
C-terminale de l'ubiquitine. Alors que les étapes de formation des conjugués et de digestion-désassemblage sont assez bien élucidées, celles qui déterminent la sélection des protéines cibles sont encore très mal connues. Il est probable que des paramètres structuraux jouent un rôle dans la reconnaissance de la cible par le conjugué ubiquitine-E2 [11].

On distingue par ailleurs de nombreuses protéines qui apparaissent ubiquitinylées de façon stable (donc non dégradées en résidus d'acides aminés mais recyclées). Ce sont, par exemple, les histones $\mathrm{H} 2 \mathrm{~A}$ et $\mathrm{H} 2 \mathrm{~B}$ dans le noyau [12], certains récepteurs membranaires comme ceux responsables de la captation des lymphocytes circulants dans les veinules post-capillaires des organes lymphoïdes [13] et l'actine.

La fonction de l'ubiquitinylation des histones dans le noyau n'est pas totalement élucidée. Il a été montré que $10 \%$ de l'histone $\mathrm{H} 2 \mathrm{~A}$ et $1 \%$ de l'histone H2B pouvaient être monoou polyubiquitinylées (portant alors des assemblages ubiquitine-ubiquitine) et que, lors de la condensation mitotique, il $y$ avait déubiquitinylation [14]. Le rôle attribué par certains auteurs à l'ubiquitine dans le maintien de la structure de la chromatine activement transcrite reste encore l'objet de controverses.

D'autres fonctions de l'ubiquitine, et non des moindres, telles que celles directement liées à la réponse au stress ou encore celles résultant de son assemblage moléculaire avec des protéines ribosomiques, sont activement étudiées [15].

Dans diverses situations pathologiques enfin, on observe une ubiquitinylation anormale d'éléments intracellulaires comme certains composés du réseau neurofibrillaire chez les patients atteints de la maladie d'Alzheimer [16]. De tels conjugués ubiquitinylés ont été caractérisés dans plusieurs maladies neurodégénératives, musculaires ou hépatiques [17].

\section{Mise en évidence et analyse de la réponse auto-immune anti-ubiquitine}

La connaissance des fonctions cellulaires multiples de l'ubiquitine et de $m / s n^{\circ} 3$, vol. 8 , mars 92

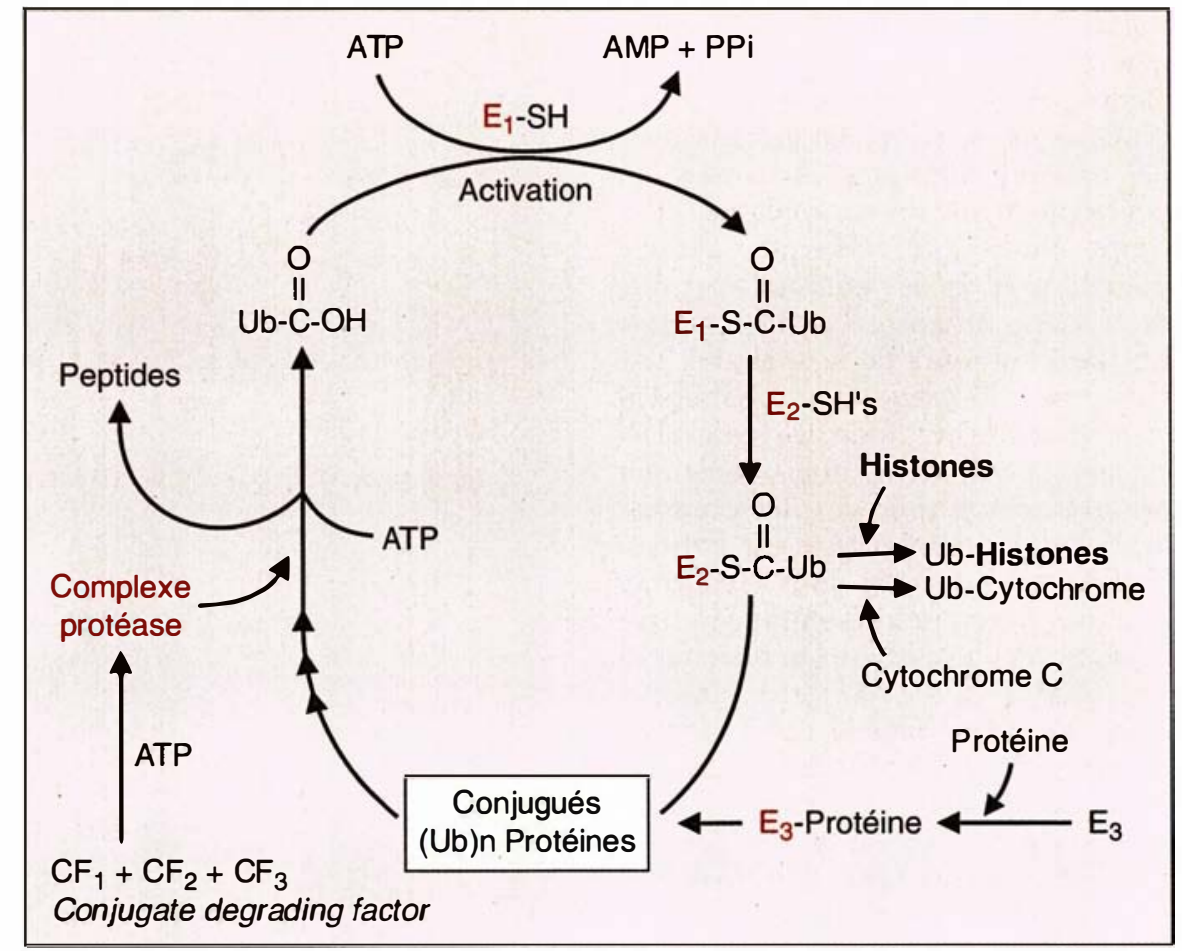

Figure 3. Cycle protéolytique dépendant de I'ubiquitine. E1 : enzyme d'activation de l'ubiquitine; E2 (famille d'enzymes) : protéines porteuses de l'ubiquitine ; E3 : enzyme de liaison de l'ubiquitine. CF1, CF2, CF3 : facteurs de dégradation des conjugués ubiquitinylés. (D'après [7].)

la diversité des protéines cibles sur lesquelles elle se conjugue nous ont conduit à rechercher l'existence d'anticorps circulants anti-ubiquitine chez les patients auto-immuns. Depuis plusieurs années, nous sommes en effet engagés dans l'analyse - en particulier à l'aide de peptides synthétiques - de la réponse antinucléaire chez des malades atteints de lupus érythémateux disséminé (LED), de polyarthrite rhumatoïde (PR), d'arthrite chronique juvénile (ACJ) et de connectivites mixtes [18-21]. Parmi les auto-anticorps caractéristiques décrits chez ces patients, notamment chez les patients lupiques, nombreux sont ceux qui sont dirigés contre des constituants, protéines ou acides nucléiques, intervenant directement dans la transcription, la réplication, la traduction ou la réparation. Ainsi l'ubiquitine représentait, de fait, un candidat potentiel intéressant à plus d'un titre.

Nos essais ont été réalisés en utilisant de l'ubiquitine purifiée d'érythrocytes de bœuf dans des tests immunoenzymatiques en phase solide de type ELISA. Nous avons ainsi montré que près de $80 \%$ des patients lupiques non sélectionnés (c'est-à-dire en phase active ou de rémission) possédaient des anticorps anti-ubiquitine [22]. Nous avons confirmé l'existence de cette réactivité en utilisant de l'ubiquitine totalement synthétique préparée par une méthode en phase solide [10] et un peptide synthétique de la protéine couvrant les résidus 22-45. Ce fragment à lui seul mime la réactivité des auto-anticorps vis-à-vis de l'ubiquitine complète dans $94 \%$ des cas. Un point intéressant de ce travail a été de constater que, dans notre série, seuls $55 \%$ des sérums renfermaient des anticorps anti-ADN natifs, marqueurs typiques du LED, contre $79 \%$ qui réagissaient avec l'ubiquitine. En outre, moins de $16 \%$ des patients atteints d'autres maladies auto-immunes rhumatismales (PR, ACJ, sclérodermie) possédaient des anticorps réagissant avec 
l'ubiquitine ou le fragment synthétique 22-45.

Cette première analyse a été suivie d'une étude séquentielle longitudinale de patients lupiques au cours du développement de la maladie [23]. Cette étude a révélé que, comme pour la plupart des auto-anticorps, le titre des anticorps anti-ubiquitine fluctuait au cours de la maladie. Les anticorps anti-ubiquitine apparaissent souvent avant les anticorps anti-ADN et pourraient, à ce titre, constituer des marqueurs précoces des poussées lupiques. Leur fréquence est particulièrement augmentée dans les formes lupiques avec atteintes cutanées, articulaires ou du système nerveux central (SNC) et s'avère très sensiblement diminuée dans le cas de formes lupiques avec détérioration rénale. Nous avons observé que d'autres auto-anticorps suivaient les mêmes évolutions (anticorps anti-histones, par exemple), alors que l'activité de certains anticorps (par exemple anti$\mathrm{Sm})$ ne variaient pas, ou peu, en fonction des formes cliniques de la maladie. Il est possible que certains auto-anticorps viennent à participer à la formation de complexes qui se déposent dans différents tissus vitaux

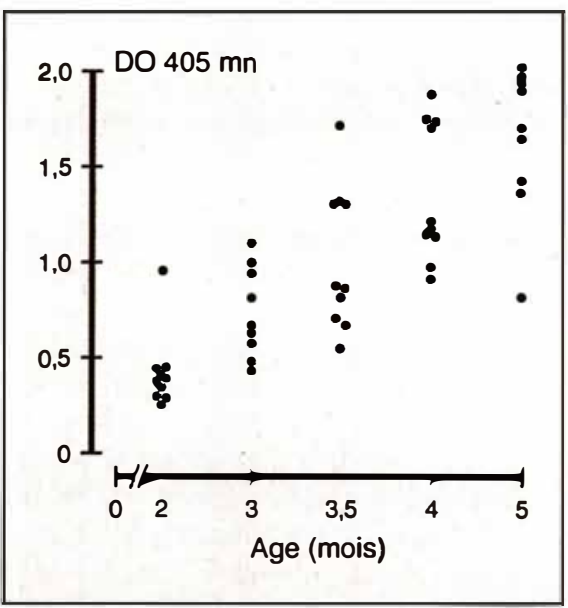

Figure 4. Apparition des anticorps de classe IgG réagissant avec le peptide 22-45 de l'ubiquitine chez la souris auto-immune MBL Ipr/lpr $\left(\boldsymbol{H}-2^{K}\right)$. Le taux d'anticorps a été mesuré en ELISA au cours du temps dans un lot de souris de 2 à 5 mois. (S. Izui, Genève, communication personnelle.)

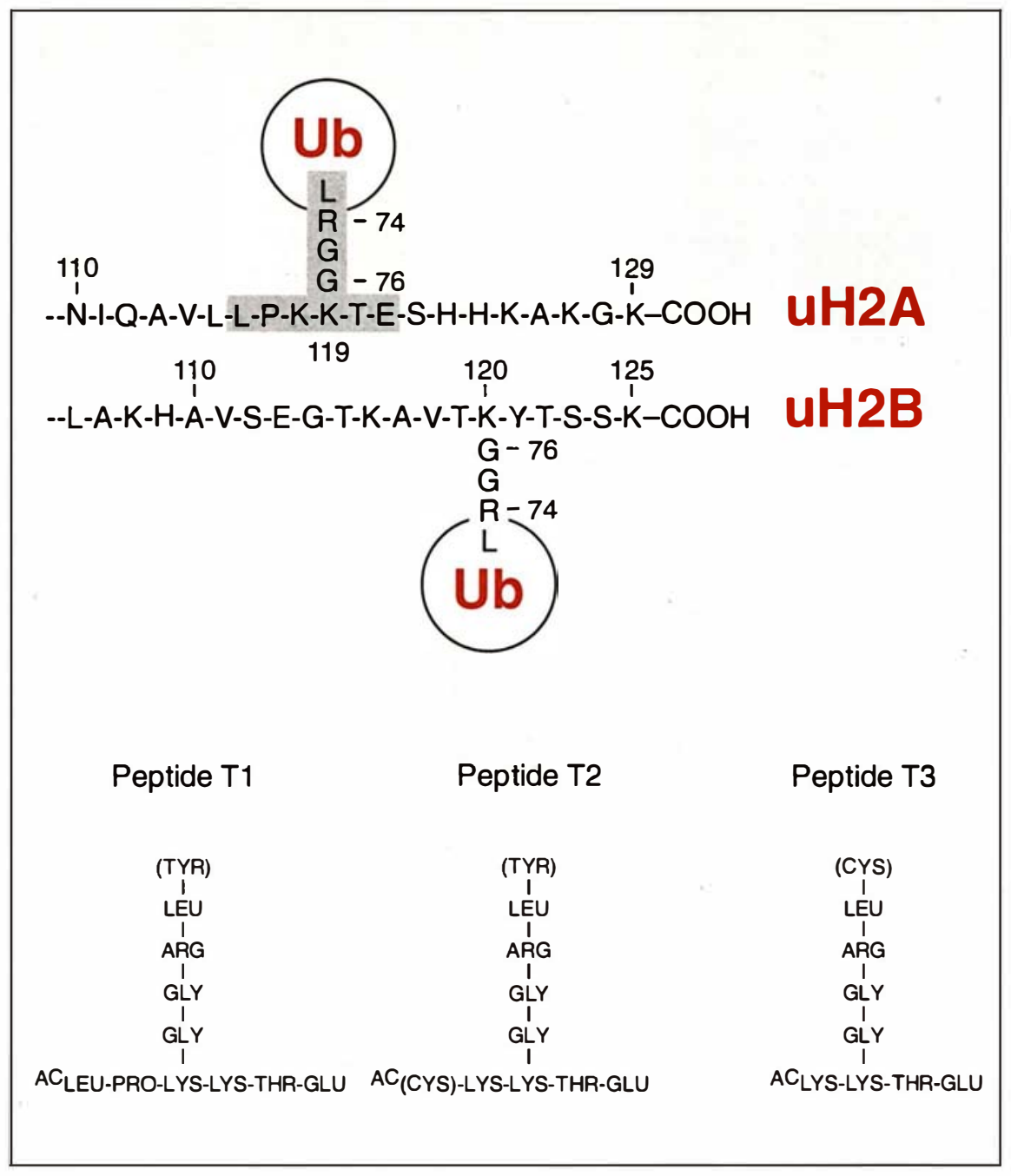

Figure 5. Régions branchées de $U$-H2A et $U$-H2B. Structure des peptides synthétiques $T 1$ à $T 3$ de U-H2A préparés pour étudier la réponse auto-immune chez les patients lupiques. (D'après [12].)

et entraînent les complications cliniques typiques que l'on décrit couramment ; le taux de ces anticorps chuterait alors de manière sensible dans la circulation périphérique.

Des modèles auto-immuns animaux sont actuellement étudiés. Ainsi, S. Izui, de l'université de Genève, a analysé la production d'auto-anticorps reconnaissant le peptide 22-45 de l'ubiquitine apparaissant au cours de la maladie auto-immune chez la souris lupique MRL lpr/lqr, qui a une espérance de vie moyenne de cinq mois (figure 4). Il a observé que, dès le $3^{\mathrm{e}}$ mois, toutes les souris étudiées produisaient des auto-anticorps reconnaissant ce peptide 22-45. L'existence de tels modèles d'étude sera très précieuse dans le futur pour analyser les événements qui lient le terrain génétique des individus à la réponse autoimmune (observations cliniques et biologiques) sans subir les retombées incontrôlables des traitements mis en œuvre chez les patients lupiques.

\section{Réponse auto-immune contre les protéines nucléaires ubiquitinylées}

Comme nous l'avons indiqué plus haut, l'ubiquitine prédomine dans la cellule sous forme conjuguée. Malheureusement, très peu de complexes ubiquitinylés ont, à ce jour, pu être 
isolés et purifiés en quantités suffisantes pour permettre de réaliser une analyse poussée de la réactivité de complexes natifs ou, par exemple, de séquencer le domaine de branchement de l'ubiquitine sur la protéine acceptrice. A notre connaissance, les régions de branchement de $\mathrm{U}-\mathrm{H} 2 \mathrm{~A}$, $\mathrm{U}-\mathrm{H} 2 \mathrm{~B}$ et de U-cytochrome C sont les seules à avoir été précisément définies à partir de ces complexes isolés in vivo (figure 5). Nous avons à nouveau utilisé l'approche peptidique pour analyser la réactivité de ces complexes covalents particuliers. Une série de peptides branchés mimant le domaine de liaison de l'ubiquitine sur H2A ont été synthétisés (figure 5) et nous avons testé en ELISA l'activité des sérums de patients à l'égard de ces sondes peptidiques [24]. Nous avons ainsi montré que les autoanticorps anti-ubiquitine présents dans le sérum de patients lupiques réagissaient particulièrement avec ces peptides sondes appelés T1, T2 et T3 : $92 \%$ des sérums de patients lupiques réagissant avec l'ubiquitine reconnaissent les peptides branchés et ces sondes peptidiques semblent surtout très spécifiques du LED puisque aucun des sérums possédant des anticorps anti-ubiquitine des patients $\mathrm{PR}$ ne réagit avec ces peptides (Tableau I). Ce point est intéressant puisqu'il suggère que la spécificité fine et donc l'origine des auto-anticorps antiubiquitine dans la PR semblent différentes de celles des anticorps antiubiquitine dans le LED.

\section{Ubiquitine et lésions tissulaires}

Chez le patient lupique, les manifestations cliniques touchant le tissu cardiaque, le SNC ou le rein sont généralement de mauvais pronostic. En particulier, et surtout au moment des poussées lupiques, des dépôts de complexes immuns peuvent être visualisés par fluorescence indirecte sur des biopsies glomérulaires. La présence de dépôts d'H2A ubiquitinylée le long de la membrane basale glomérulaire a été mise en évidence en utilisant des anticorps anti-peptide $\mathrm{T}$ très spécifiques de U-H2A, qui ne réagissent ni avec l'ubiquitine libre ni avec H2A libre (figure 6, p. 231). Ces dépôts d'U-H2A peuvent résulter de $m / s n^{\circ} 3$, vol. 8, mars 92

Tableau I.

RÉACTIVITÉ EN ELISA DES SÉRUMS DE PATIENTS LUPIQUES ET PR AVEC LE PEPTIDE SYNTHÉTIQUE T2 de U-H2A

\begin{tabular}{|c|c|c|}
\hline Sérums & $\begin{array}{c}\text { Anticorps } \\
\text { anti-ubiquitine }\end{array}$ & Fréquence de positivité/peptide T2 \\
\hline LED & $\begin{array}{l}+ \\
-\end{array}$ & $\begin{array}{r}36 / 39(92 \%) \\
2 / 15(13 \%)\end{array}$ \\
\hline PR & $\begin{array}{l}+ \\
-\end{array}$ & $\begin{array}{ll}0 / 7 & (0) \\
0 / 14 & (0)\end{array}$ \\
\hline
\end{tabular}

Résultats partiels d'après Plaué et al. [24]. On observe que $13 \%$ des sérums de patients lupiques sont ubiquitine $-\pi 2^{+}$.

deux mécanismes distincts qu'il nous faudra analyser plus avant. Il est possible d'imaginer en effet que l'H2A ubiquitinylée, tout comme les autres histones [25], se lie directement aux constituants de la membrane basale. U-H2A serait alors soit isolée, soit partie intégrante d'une structure nucléosomique qui semble jouer un rôle clé dans le schéma lésionnel actuellement proposé [26]. A l'inverse, on peut aussi avancer un système différent dans lequel l'ubiquitinylation de $\mathrm{H} 2 \mathrm{~A}$ interviendrait dans une étape secondaire, suite à l'inflammation provoquée par le dépôt de complexes immuns et le déclenchement du syndrome néphrotique.

\section{Place des protéines nucléaires ubiquitinylées dans la réponse auto-immune}

De nombreux travaux ont traité du rôle des protéines de choc thermique (HSP) ou d'autres protéines de stress dans différents mécanismes immuns ( $\mathrm{m} / \mathrm{s} n^{\circ} 1$, vol. 6, p. 80) [2, 27]. En particulier, elles seraient impliquées dans des mécanismes auto-immuns et dans la pathogenèse de l'arthrite. Les HSP sont à la fois très conservées et immunogènes. Ces propriétés pourraient expliquer que des protéines de stress présentes chez divers microorganismes infectieux puissent déclencher une réponse humorale et cellulaire auto-immune et l'expression d'atteintes auto-immunes. Les protéines de stress de Mycobacterium tuberculosis ou d'autres bactéries sont très voisines des protéines de stress des mammifères et pourraient être impliquées dans la pathogenèse de l'arthrite induite par de l'adjuvant chez le rat et peut-être dans la PR humaine. Elles interviendraient ainsi dans les phénomènes auto-immuns par un processus de mimétisme moléculaire. Par exemple, on peut citer l'existence chez les patients lupiques d'autoanticorps anti-HSP 90 et HSP 70 et celle d'auto-anticorps anti-HSP 65 chez des malades atteints de PR [28, 29].

Il est très vraisemblable que ce mécanisme de mimétisme que nous venons d'évoquer pour les HSP de hauts poids moléculaires n'entre pas en jeu dans le cas de l'ubiquitine et de UH2A. En effet, l'ubiquitine n'est pas présente dans les bactéries ou les virus et ne peut donc être à l'origine d'anticorps doués de réactivité croisée comme on le décrit dans le cas des autres HSP. De même, les histones sont des protéines nucléaires typiquement eucaryotiques qui sont remplacées dans les bactéries par des protéines chromosomales fonctionnellement mais non structuralement reliées [30]. La réponse auto-immune dirigée contre $\mathrm{U}-\mathrm{H} 2 \mathrm{~A}$ relèverait donc d'un processus différent de celui du mimétisme moléculaire.

Plusieurs mécanismes non exclusifs peuvent être invoqués pour rendre 


\section{RÉFÉRENCES}

25. Schmiedecke TMJ, Stöckl FW, Weber R, Sugisaki Y, Batsford SR, Vogt A. Histones have high affinity for the glomerular bascment membranc. Relevance for immune complex formation in lupus nephritis. $J$ Exp Med 1989 ; 169 : 1879-94.

26. Brinkman K, Termaat RM, Berden JHM, Smeenk RJT. Anti-DNA antibodies and lupus nephritis: the complexity of crossrcactivity. Immunol Today 1990; 11 : 232-4.

27. Kaufmann SHE. Heat shock protcins and the immune response. Immunol Today $1990 ; 11: 129-36$

28. Norton PM, Iscnberg DA, Latchman DS. Elevated levels of the $90 \mathrm{Kd}$ heat shock protcin in a proportion of SLE patients with active disease. J Autoimm $1989 ; 2$ : 187-95.

29. Tsoulfa G, Rook GAW, Bahr GM, et al. Elevated IgG antibody levels to the mycobacterial $65 \mathrm{kDa}$ heat shock protein are characteristic of patients with rheumatoid arthritis. Scand J Immunol 1989 ; 30 : 519-27.

30. Laine B, Chartier F, Imbert M, Sautière $\mathrm{P}$. Structural and functional propertics of the chromosomal protein $\mathrm{MC} 1$ isolated from various strains of methano sarcinaceac. In : Belaich JP, et al., cds. New York : Plcnum Press, 1990 : 291-301.

31. Atanassov C, Briand JP, Bonnicr D, Van Regenmortel MHV, Muller S. New Zcaland whitc rabbits immunized with RNA-complexed total histones develop an auto-immunc-like response. Clin Exp Immunol 1991; 86 : 124-33.

32. Hardin JA. The lupus auto-antigens and the pathogenesis of systemic lupus crythematosus. Arthritis Rheum 1986; 29 . 457-60.

33. Fournié G. Circulating DNA and lupus nephritis. Kidney Int 1988 ; 33 : 487-97.

34. Bell DA, Morrison B, Vanden-Bygaart P. Immunogenic DNA-related factors. Nucleosomes spontaneously released from normal murine lymphoid cells stimulate proliferation and immunoglobulin synthesis of normal mouse lymphocytes. J Clin Invest $1990 ; 85: 1487-96$.

35. Pechan PM. Heat shock proteins and cell proliferation. FEBS Lett 1991; 280 : 1-4.

36. Zouali M, Fournié J, Thèzc J. Quantitative clonal analysis of the B-cell repertoire in human lupus. Cell Immunol 1991 ; $133: 161-77$.

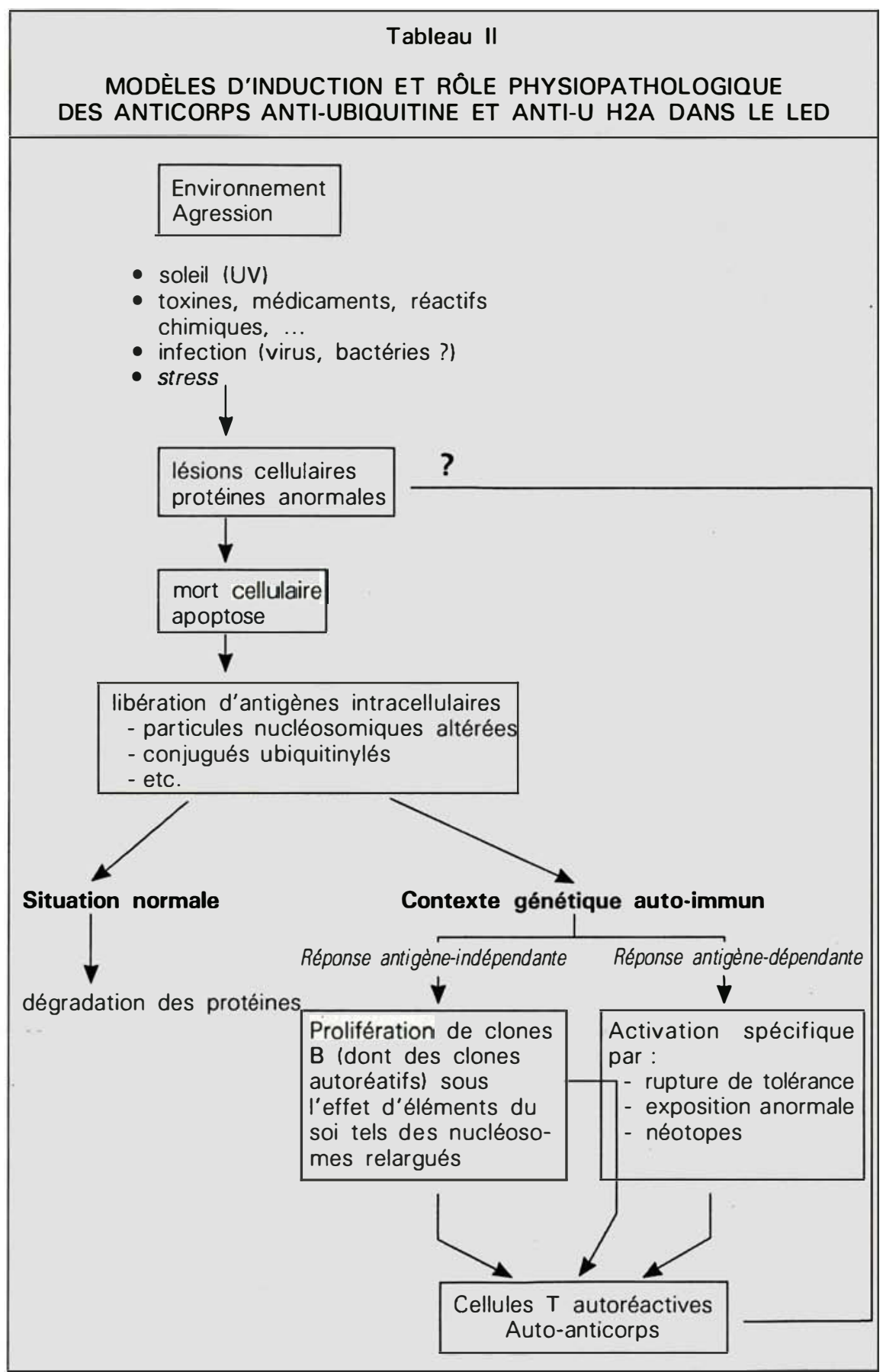

compte de la production d'anticorps anti-U-H2A (Tableau II). Dans les voies possibles que nous proposons, la mort cellulaire par apoptose et la libération d'éléments intracellulaires ubiquitinylés, ou non, sont au centre du mécanisme inducteur. La cause de l'accumulation des conjugués ubiquitinylés demeure encore obscure. D. Latchman, à Londres, a récem- ment montré que la transcription des gènes qui codent pour l'ubiquitine n'est pas augmentée chez les patients lupiques alors que celle des gènes codant pour les HSP 90 et 70 était surexprimée dans les cellules mononucléées du sang périphérique de ces malades (communication personnelle). Il faut dès lors considérer d'autres causes d'accumulation, par exemple 


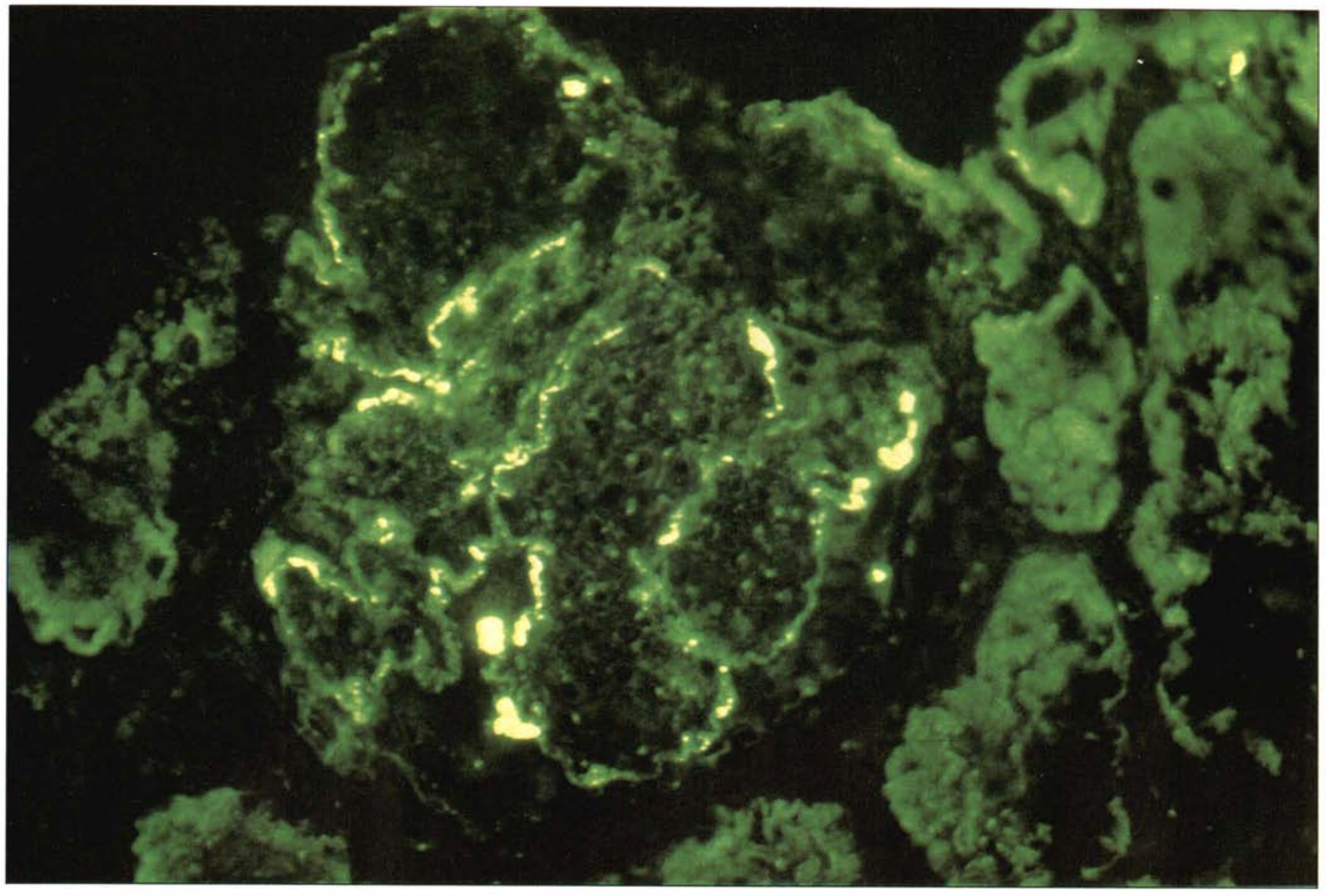

Figure 6. Dépôts d'U-H2A révélés par immunofluorescence à l'aide d'anticorps anti-peptide T2 le long des capillaires glomérulaires et dans le mésangium. Biopsie de rein (section de $4 \mu \mathrm{m}$ ) d'un patient lupique présentant une néphrite membrano-proliférative de type IV. (F. Stöckl, Fribourg, communication personnelle.)

par suite d'erreurs métaboliques, en particulier cataboliques, qui interviendraient dans le schéma présenté sur la figure 3, ou lors d'une libération importante de complexes ubiquitinylés par mort cellulaire excessive ou accélérée. Il est intéressant de replacer dans ce contexte des résultats récents que nous avons obtenus en utilisant un modèle animal de lapins New Zealand [31].

Nous avons établi que de tels lapins immunisés par un mélange d'histones pures associées à de l'ARN comme élément transporteur (D. Stollar a montré en 1970 que l'adjonction d'ADN ou d'ARN à des histones rendait ces protéines immunogènes) répondaient par la production de toute une série d'anticorps de classe IgG de spécificités très proches de celles que l'on peut caractériser chez les patients lupiques. En particulier, outre la réponse anti-histones, nous avons observé une réponse précoce anti-ubiquitine et anti-U-H2A, puis une réponse plus tardive anti-ADN Ce type de réactivité n'a pas été observé après l'immunisation d'animaux soit par des nucléosomes intacts, soit par le mélange d'histones sans ARN, soit encore par l'une quelconque des histones $\mathrm{H} 1, \mathrm{H} 2 \mathrm{~A}$, $\mathrm{H} 2 \mathrm{~B}, \mathrm{H} 3$ et $\mathrm{H} 4$ isolées. Certains des lapins immunisés ont présenté une perte de poids progressive accompagnée d'une diminution très sensible du tonus musculaire et de la mort précoce. Des études complémentaires sont en cours visant à étudier les dépôts d'immunoglobulines dans les capillaires glomérulaires et dans les aires mésangiales des reins avec une éventuelle glomérulonéphrite. Globalement, ces résultats peuvent s'interpréter comme reflétant une réponse immune induite par une structure nucléosomique altérée dans un contexte auto-immun prolifératif et de mort cellulaire (Tableau II). Nous noterons par ailleurs que ces observations soutiennent l'hypothèse de plus en plus acceptée selon laquelle, dans le lupus, la réponse autoimmune serait induite par un antigène multimoléculaire, en particulier la structure nucléosomique, plutôt que par un constituant isolé $[18,32$, 33].

Que ce soit du fait de l'accumulation anarchique de conjugués ubiquitinylés par défaut métabolique ou par mort cellulaire, ces complexes peuvent intervenir à différents niveaux de la réponse auto-immune (Tableau II). Tout d'abord, ils peuvent orienter une réponse spécifique de l'antigène. Les propriétés immunogènes de tels complexes peuvent s'expliquer de dif- 
férentes manières, par exemple par une rupture de tolérance du fait d'une délocalisation ou d'un eff et de masse. Il est aussi possible que l'ubiquitinylation joue un rôle déterminant de médiateur de la réponse auto-immune en modifiant la configuration des différents composants des conjugués qui s'accumulent, créant ainsi de nouveaux épitopes (néotopes).

Le deuxième niveau où la libération d'antigènes intracellulaires multimoléculaires peut exercer un rôle central concerne leur rôle direct sur la prolifération de clones de lymphocytes $\mathrm{B}$. Ce phénomène a récemment été décrit par Bell et al. [34] qui ont examiné l'effet immunoprolifératif de nucléosomes. L'ubiquitine semble aussi jouer un rôle clé dans la croissance et la prolifération cellulaires [35]. Ainsi, les mêmes structures relarguées du contenu cellulaire et éventuellement ubiquitinylées pourraient intervenir de manière décisive à la fois dans un schéma prolifératif de type activation polyclonale non spécifique et lors d'une première activation de clones B suivie d'étapes d'activation spécifique. Récemment, Zouali et al. ont analysé de manière quantitative le répertoire cellulaire $B$ d'individus normaux et de patients lupiques [36]. Ils ont notamment montré l'existence, chez les seuls patients lupiques, de cellules $B$ productrices d'anticorps de classe IgG réagissant avec l'ADN, le poly (dT), l'antigène Sm et l'ubiquitine. On peut parallèlement imaginer une réponse immune spécifique contre une structure nucléosomique altérée qui induirait par ailleurs des clones $\mathrm{T}$ autoréactifs. Enfin, l'effet prolifératif peut aussi s'exercer sur des lymphocytes B autoréactifs dont on sait qu'ils sont largement représentés dans le répertoire [36]. Du fait de la participation d'éléments multimoléculaires, on peut admettre qu'il puisse y avoir une réponse contre les éléments prolifératifs eux-mêmes et contre les structures qui leur sont associées - par exemple, dans le cas des nucléosomes, l'ADN, les histones ; des HMG (high mobility group proteins), les ARN polymérases, etc. - certains étant la cible d'une reconnaissance $B$, d'autres celle d'une reconnaissance $T$. Il est évident que ce schéma représente un modèle d'étude encore hypothéti- devront étayer. Il a cependant le mérite de considérer un certain nombre d'observations apparemment indépendantes qu'il est souvent difficile aujourd'hui de concilier, par exemple la multiplicité de réponses humorales, la prolifération lymphocytaire et le fait que, paradoxalement, les événements dans le lupus peuvent être permanents (du fait de la dépendance génétique par exemple) et soumis à des exacerbations imprévisibles (par exemple liées au stress). Ce schéma n'explique certainement pas tout et, en l'occurrence, l'éventualité d'un rôle physiopathologique des anticorps antiubiquitine n'est pas démontré. Il faut souligner que cette question a été rarement résolue dans le cas des autoanticorps typiques des maladies autoimmunes non spécifiques d'organes. Il faudra également tenter de déterminer si la réponse anti-ubiquitine joue un rôle initial ou si elle est une conséquence plus tardive de l'état autoimmun (inflammatoire) en général. Dans l'avenir, une meilleure convergence des connaissances entre, d'une part, les études fondamentales sur les mécanismes auto-immunitaires (par exemple, à l'aide de modèles lupiques murins) et, d'autre part, les voies métaboliques du système ubiquitine et ses dysfonctionnements, sera très utile pour modifier ou renforcer la validité de ce modèle. Déjà, les efforts sont portés sur les phénomènes de réparation cellulaire qui sont particulièrement sollicités lors de l'établissement du terrain inflammatoire typique du LED et où l'ubiquitine, de par ses fonctions, pourrait jouer un rôle majeur

\section{Remerciements}

Je voudrais remercier S. Izui (Genève) D. Latchman (Londres) et F. Stöckl (Fribourg, Allemagne) pour les résultats non publiés qu'il m'ont confiés. Je suis particulièrement reconnaissante à $\mathrm{K}$. Hannestad (Troms $\phi$, Norvège) et G. Fournié (Toulouse) pour leur lecture critique du manuscrit. Je remercie également mes collègues qui ont participé à l'obtention de ces résultats, J.-P. Briand, D.-A. Isenberg et S. Plaué, ainsi que M.H.V. Van Regenmortel, dans le laboratoire duquel ce travail a été réalisé. Je tiens à remercier enfin S. Wencker pour la préparation de ce manuscrit.

\section{Summary}

Place and role of ubiquitin in autoimmunity development in systemic lupus erythematous

Etiologic factors involved in organ non-specific autoimmune diseases (such as systemic lupus erythematosus (SLE), rheumatoid arthritis or Gougerot-Sjögren's syndrome) are far from being elucidated. Several prominent hypothesis have been proposed but alone they cannot reconcile all clinical and experimental data described in the literature. For example, the existence of - a B-cell hyper-responsiveness ground does exist with a possible determining role of some autoreactive $B$ and $T$ lymphocyte subpopulations. A disequilibrium of $\mathrm{T}$ lymphocyte sub-populations certainly contributes to the autoimmune state. These last years, it has become increasingly clear that stress proteins (HSPs) play a role in various immune mechanisms. In the pathogenesis of SLE, one of these HSPs, namely ubiquitin, seems to take a major place. Among its various functions, ubiquitin is involved in intracellular proteolysis of altered protein, in the chromatin and ribosome structure and in the lymphocyte homing. The recent finding that circulating autoantibodies reacting whith ubiquitin and overall, with ubiquitinated histone $\mathrm{H} 2 \mathrm{~A}$, do exist in the serum of patients with SLE indicates that the ubiquitin system could be involved in autoantibody induction within a predisposed genetic context, and thus play a major role in SLE. In this process, cell death and repair events probably hold a prominent place.

\section{TIRÉS A PART}

S. Muller. 